\title{
New General Integral Inequalities for Lipschitzian Functions via Hadamard Fractional Integrals
}

\author{
İmdat İşcan \\ Department of Mathematics, Faculty of Arts and Sciences, Giresun University, 28100 Giresun, Turkey \\ Correspondence should be addressed to İmdat İşcan; imdat.iscan@giresun.edu.tr \\ Received 13 January 2014; Revised 18 March 2014; Accepted 1 April 2014; Published 22 April 2014 \\ Academic Editor: Julien Salomon
}

Copyright (C) 2014 İmdat İşcan. This is an open access article distributed under the Creative Commons Attribution License, which permits unrestricted use, distribution, and reproduction in any medium, provided the original work is properly cited.

The author obtains new estimates on generalization of Hadamard, Ostrowski, and Simpson type inequalities for Lipschitzian functions via Hadamard fractional integrals. Some applications to special means of positive real numbers are also given.

\section{Introduction}

Let real function $f$ be defined on some nonempty interval $I$ of real line $\mathbb{R}$. The function $f$ is said to be convex on $I$ if inequality

$$
f(t x+(1-t) y) \leq t f(x)+(1-t) f(y)
$$

holds for all $x, y \in I$ and $t \in[0,1]$.

Following are inequalities which are well known in the literature as Hermite-Hadamard inequality, Ostrowski inequality, and Simpson inequality, respectively.

Theorem 1. Let $f: I \subseteq \mathbb{R} \rightarrow \mathbb{R}$ be a convex function defined on the interval I of real numbers and $a, b \in I$ with $a<b$. The following double inequality holds:

$$
f\left(\frac{a+b}{2}\right) \leq \frac{1}{b-a} \int_{a}^{b} f(x) d x \leq \frac{f(a)+f(b)}{2} .
$$

Theorem 2. Let $f: I \subseteq \mathbb{R} \rightarrow \mathbb{R}$ be a mapping differentiable in $I^{\circ}$, the interior of $I$, and let $a, b \in I^{\circ}$ with $a<b$. If $\left|f^{\prime}(x)\right| \leq$ $M, x \in[a, b]$; then the following inequality holds:

$$
\left|f(x)-\frac{1}{b-a} \int_{a}^{b} f(t) d t\right| \leq \frac{M}{b-a}\left[\frac{(x-a)^{2}+(b-x)^{2}}{2}\right]
$$

for all $x \in[a, b]$.
Theorem 3. Let $f:[a, b] \rightarrow \mathbb{R}$ be four times continuously differentiable mapping on $(a, b)$ and $\left\|f^{(4)}\right\|_{\infty}=$ $\sup _{x \in(a, b)}\left|f^{(4)}(x)\right|<\infty$. Then the following inequality holds:

$$
\begin{gathered}
\left|\frac{1}{3}\left[\frac{f(a)+f(b)}{2}+2 f\left(\frac{a+b}{2}\right)\right]-\frac{1}{b-a} \int_{a}^{b} f(x) d x\right| \\
\leq \frac{1}{2880}\left\|f^{(4)}\right\|_{\infty}(b-a)^{4} .
\end{gathered}
$$

In recent years, many authors have studied errors estimations for Hermite-Hadamard, Ostrowski, and Simpson inequalities; for refinements, counterparts, and generalization, see [1-9] and references therein.

The following definitions are well known in the literature.

Definition 4. A function $f: I \subseteq \mathbb{R} \rightarrow \mathbb{R}$ is called an $M$ Lipschitzian function on the interval $I$ of real numbers with $M \geq 0$ if

$$
|f(x)-f(y)| \leq M|x-y|
$$

for all $x, y \in I$.

For some recent results connected with HermiteHadamard type integral inequalities for Lipschitzian functions, see [10-13]. 
Definition 5 (see $[14,15])$. A function $f: I \subseteq(0, \infty) \rightarrow \mathbb{R}$ is said to be GA-convex (geometric arithmetically convex) if

$$
f\left(x^{t} y^{1-t}\right) \leq t f(x)+(1-t) f(y)
$$

for all $x, y \in I$ and $t \in[0,1]$.

We will now give definitions of the right-sided and left-sided Hadamard fractional integrals which are used throughout this paper.

Definition 6. Let $f \in L[a, b]$. The right-sided and left-sided Hadamard fractional integrals $J_{a^{+}}^{\alpha} f$ and $J_{b^{-}}^{\alpha} f$ of oder $\alpha>0$ with $b>a \geq 0$ are defined by

$$
\begin{aligned}
& J_{a+}^{\alpha} f(x)=\frac{1}{\Gamma(\alpha)} \int_{a}^{x}\left(\ln \frac{x}{t}\right)^{\alpha-1} f(t) \frac{d t}{t}, \quad a<x<b, \\
& J_{b-}^{\alpha} f(x)=\frac{1}{\Gamma(\alpha)} \int_{x}^{b}\left(\ln \frac{t}{x}\right)^{\alpha-1} f(t) \frac{d t}{t}, \quad a<x<b,
\end{aligned}
$$

respectively, where $\Gamma(\alpha)$ is Gamma function defined by $\Gamma(\alpha)=\int_{0}^{\infty} e^{-t} t^{\alpha-1} d t($ see $[16])$.

In [17], Iscan established Hermite-Hadamard's inequalities for GA-convex functions in Hadamard fractional integral forms as follows.

Theorem 7. Let $f: I \subseteq(0, \infty) \rightarrow \mathbb{R}$ be a function such that $f \in L[a, b]$, where $a, b \in I$ with $a<b$. If $f$ is $a$ GA-convex function on $[a, b]$, then the following inequalities for fractional integrals hold:

$$
\begin{aligned}
f(\sqrt{a b}) & \leq \frac{\Gamma(\alpha+1)}{2(\ln (b / a))^{\alpha}}\left\{J_{a+}^{\alpha} f(b)+J_{b-}^{\alpha} f(a)\right\} \\
& \leq \frac{f(a)+f(b)}{2}
\end{aligned}
$$

with $\alpha>0$.

In the inequality (8), if we take $\alpha=1$, then we have the following inequality:

$$
f(\sqrt{a b}) \leq \frac{1}{\ln b-\ln a} \int_{a}^{b} \frac{f(t)}{t} d t \leq \frac{f(a)+f(b)}{2} .
$$

Morever, in [17], Iscan obtained a generalization of Hadamard, Ostrowski, and Simpson type inequalities for quasigeometrically convex functions via Hadamard fractional integrals as related to the inequality (8).

In this paper, the author obtains new general inequalities for Lipschitzian functions via Hadamard fractional integrals as related to the inequality (8).

\section{Main Results}

Let $f: I \subseteq(0, \infty) \rightarrow \mathbb{R}$ be a $M$-Lipschitzian function on $I$; throughout this section, we will take

$$
\begin{aligned}
I_{f}(x, \lambda, \alpha, a, b)= & (1-\lambda)\left[\ln ^{\alpha} \frac{x}{a}+\ln ^{\alpha} \frac{b}{x}\right] f(x) \\
& +\lambda\left[f(a) \ln ^{\alpha} \frac{x}{a}+f(b) \ln ^{\alpha} \frac{b}{x}\right] \\
& -\Gamma(\alpha+1)\left[J_{x-}^{\alpha} f(a)+J_{x+}^{\alpha} f(b)\right], \\
S_{f}(x, y, \lambda, \alpha, a, b)= & \lambda^{\alpha} f(x)+(1-\lambda)^{\alpha} f(y) \\
& -\frac{\Gamma(\alpha+1)}{\ln ^{\alpha}(b / a)}\left[J_{C-}^{\alpha} f(a)+J_{C+}^{\alpha} f(b)\right],
\end{aligned}
$$

where $a, b \in I$ with $a<b, x, y \in[a, b], \lambda \in[0,1], C=a^{1-\lambda} b^{\lambda}$, $\alpha>0$ and $\Gamma$ is Euler Gamma function.

Theorem 8. Let $f: I \subseteq(0, \infty) \rightarrow \mathbb{R}$ be a M-Lipschitzian function on $I$ and $a, b \in I$ with $a<b$. Then for all $x \in[a, b]$, $\lambda \in[0,1]$, and $\alpha>0$, we have the following inequality for Hadamard fractional integrals:

$$
\begin{aligned}
\left|I_{f}(x, \lambda, \alpha, a, b)\right| & \\
\leq M & \left\{[(1-\lambda) x-\lambda a]\left(\ln \frac{x}{a}\right)^{\alpha}+\alpha(2 \lambda-1)\right. \\
& \times \int_{a}^{x}\left(\ln \frac{t}{a}\right)^{\alpha-1} d t+[\lambda b-(1-\lambda) x] \\
& \left.\times\left(\ln \frac{b}{x}\right)^{\alpha}+\alpha(1-2 \lambda) \int_{x}^{b}\left(\ln \frac{b}{t}\right)^{\alpha-1} d t\right\} .
\end{aligned}
$$

Proof. Using the hypothesis of $f$, we have the following inequality:

$$
\begin{aligned}
& \left|I_{f}(x, \lambda, \alpha, a, b)\right| \\
& =\mid(1-\lambda)\left[\ln ^{\alpha} \frac{x}{a}+\ln ^{\alpha} \frac{b}{x}\right] f(x) \\
& +\lambda\left[f(a) \ln ^{\alpha} \frac{x}{a}+f(b) \ln ^{\alpha} \frac{b}{x}\right] \\
& \quad-\alpha\left[\int_{a}^{x}\left(\ln \frac{t}{a}\right)^{\alpha-1} f(t) \frac{d t}{t}+\int_{x}^{b}\left(\ln \frac{b}{t}\right)^{\alpha-1} f(t) \frac{d t}{t}\right] \mid \\
& \leq(1-\lambda) \mid f(x) \ln ^{\alpha} \frac{x}{a}-\alpha \int_{a}^{x}\left(\ln \frac{t}{a}\right)^{\alpha-1} f(t) \frac{d t}{t} \\
& +f(x) \ln ^{\alpha} \frac{b}{x}-\alpha \int_{x}^{b}\left(\ln \frac{b}{t}\right)^{\alpha-1} f(t) \frac{d t}{t} \mid
\end{aligned}
$$


International Journal of Analysis

3

$$
\begin{aligned}
& +\lambda \mid f(a) \ln ^{\alpha} \frac{x}{a}-\alpha \int_{a}^{x}\left(\ln \frac{t}{a}\right)^{\alpha-1} f(t) \frac{d t}{t} \\
& +f(b) \ln ^{\alpha} \frac{b}{x}-\alpha \int_{x}^{b}\left(\ln \frac{b}{t}\right)^{\alpha-1} f(t) \frac{d t}{t} \\
& \leq \alpha(1-\lambda)\left[\int_{a}^{x}\left(\ln \frac{t}{a}\right)^{\alpha-1}|f(x)-f(t)| \frac{d t}{t}\right. \\
& \left.+\int_{x}^{b}\left(\ln \frac{b}{t}\right)^{\alpha-1}|f(x)-f(t)| \frac{d t}{t}\right] \\
& +\alpha \lambda\left[\int_{a}^{x}\left(\ln \frac{t}{a}\right)^{\alpha-1}|f(a)-f(t)| \frac{d t}{t}\right. \\
& \left.+\int_{x}^{b}\left(\ln \frac{b}{t}\right)^{\alpha-1}|f(b)-f(t)| \frac{d t}{t}\right] \\
& \leq \alpha(1-\lambda) M\left[\int_{a}^{x}\left(\ln \frac{t}{a}\right)^{\alpha-1}(x-t) \frac{d t}{t}\right. \\
& \left.+\int_{x}^{b}\left(\ln \frac{b}{t}\right)^{\alpha-1}(t-x) \frac{d t}{t}\right] \\
& +\alpha \lambda M\left[\int_{a}^{x}\left(\ln \frac{t}{a}\right)^{\alpha-1}(t-a) \frac{d t}{t}\right. \\
& \left.+\int_{x}^{b}\left(\ln \frac{b}{t}\right)^{\alpha-1}(b-t) \frac{d t}{t}\right] \\
& \leq M\left\{[(1-\lambda) x-\lambda a]\left(\ln \frac{x}{a}\right)^{\alpha}\right. \\
& +\alpha(2 \lambda-1) \int_{a}^{x}\left(\ln \frac{t}{a}\right)^{\alpha-1} d t \\
& +[\lambda b-(1-\lambda) x]\left(\ln \frac{b}{x}\right)^{\alpha} \\
& \left.+\alpha(1-2 \lambda) \int_{x}^{b}\left(\ln \frac{b}{t}\right)^{\alpha-1} d t\right\} .
\end{aligned}
$$

Corollary 9. In Theorem 8, If we take $\lambda=0$, then we get

$$
\begin{aligned}
& \mid \frac{(\ln (x / a))^{\alpha}+(\ln (b / x))^{\alpha}}{(\ln (b / a))^{\alpha}} f(x)-\frac{\Gamma(\alpha+1)}{(\ln (b / a))^{\alpha}} \\
& \times\left[J_{x-}^{\alpha} f(a)+J_{x+}^{\alpha} f(b)\right] \mid \\
& \leq \frac{M}{(\ln (b / a))^{\alpha}} \\
& \quad \times\left\{x\left[\left(\ln \frac{x}{a}\right)^{\alpha}-\left(\ln \frac{b}{x}\right)^{\alpha}\right]\right. \\
& \left.\quad+\alpha\left[\int_{x}^{b}\left(\ln \frac{b}{t}\right)^{\alpha-1} d t-\int_{a}^{x}\left(\ln \frac{t}{a}\right)^{\alpha-1} d t\right]\right\} .
\end{aligned}
$$

In this inequality,

(i) if we take $\alpha=1$, then

$$
\begin{aligned}
\mid f(x) & -\frac{1}{\ln b-\ln a} \int_{a}^{b} \frac{f(t)}{t} d t \mid \\
& \leq \frac{M}{\ln b-\ln a}\left\{x \ln \frac{x^{2}}{a b}+(a+b-2 x)\right\} .
\end{aligned}
$$

(ii) If we take $x=\sqrt{a b}$, then

$$
\begin{aligned}
\left|f(\sqrt{a b})-\frac{2^{\alpha-1} \Gamma(\alpha+1)}{(\ln (b / a))^{\alpha}}\left[J_{\sqrt{a b}-}^{\alpha} f(a)+J_{\sqrt{a b+}}^{\alpha} f(b)\right]\right| \\
\leq \frac{2^{\alpha-1} M \alpha}{(\ln (b / a))^{\alpha}} \\
\quad \times\left\{\int_{\sqrt{a b}}^{b}\left(\ln \frac{b}{t}\right)^{\alpha-1} d t-\int_{a}^{\sqrt{a b}}\left(\ln \frac{t}{a}\right)^{\alpha-1} d t\right\} .
\end{aligned}
$$

(iii) If we take $x=\sqrt{a b}$ and $\alpha=1$, then

$$
\begin{array}{r}
\left|f(\sqrt{a b})-\frac{1}{\ln b-\ln a} \int_{a}^{b} \frac{f(t)}{t} d t\right| \\
\leq \frac{M}{\ln b-\ln a}(a+b-2 \sqrt{a b}) .
\end{array}
$$

Corollary 10. In Theorem 8 , if we take $\lambda=1$, then we get

$$
\begin{aligned}
& \mid\left[\frac{f(a) \ln ^{\alpha}(x / a)+f(b) \ln ^{\alpha}(b / x)}{(\ln (b / a))^{\alpha}}\right] \\
& -\frac{2^{\alpha-1} \Gamma(\alpha+1)}{(\ln (b / a))^{\alpha}}\left[J_{x-}^{\alpha} f(a)+J_{x+}^{\alpha} f(b)\right] \mid \\
& \leq \frac{M}{(\ln (b / a))^{\alpha}} \\
& \quad \times\left\{b \ln ^{\alpha} \frac{x}{a}-a \ln ^{\alpha} \frac{b}{x}\right. \\
& \left.\quad-\alpha\left[\int_{x}^{b}\left(\ln \frac{b}{t}\right)^{\alpha-1} d t-\int_{a}^{x}\left(\ln \frac{t}{a}\right)^{\alpha-1} d t\right]\right\} .
\end{aligned}
$$

In this inequality, if we take $x=\sqrt{a b}$, then

$$
\begin{aligned}
&\left|\frac{f(a)+f(b)}{2}-\frac{2^{\alpha-1} \Gamma(\alpha+1)}{(\ln (b / a))^{\alpha}}\left[J_{\sqrt{a b}-}^{\alpha} f(a)+J_{\sqrt{a b+}}^{\alpha} f(b)\right]\right| \frac{2^{\alpha-1} M}{(\ln (b / a))^{\alpha}} \\
& \quad \times\left\{\frac{b-a}{2^{\alpha}}\left(\ln \frac{b}{a}\right)^{\alpha}\right. \\
&\left.-\alpha\left[\int_{\sqrt{a b}}^{b}\left(\ln \frac{b}{t}\right)^{\alpha-1} d t-\int_{a}^{\sqrt{a b}}\left(\ln \frac{t}{a}\right)^{\alpha-1} d t\right]\right\} .
\end{aligned}
$$

(18) 
Specially if we take $\alpha=1$ in this inequality, then we have

$$
\begin{aligned}
& \left|\frac{f(a)+f(b)}{2}-\frac{1}{\ln b-\ln a} \int_{a}^{b} \frac{f(t)}{t} d t\right| \\
& \quad \leq \frac{M}{\ln b-\ln a}\left\{\frac{b-a}{2}\left(\ln \frac{b}{a}\right)-(a+b-2 \sqrt{a b})\right\} .
\end{aligned}
$$

\section{Corollary 11. In Theorem 8 ,}

(1) if we take $x=\sqrt{a b}$ and $\lambda=1 / 3$, then

$$
\begin{aligned}
& \mid \frac{1}{3}\left[\frac{f(a)+f(b)}{2}+2 f(\sqrt{a b})\right] \\
& -\frac{2^{\alpha-1} \Gamma(\alpha+1)}{(\ln (b / a))^{\alpha}}\left[J_{\sqrt{a b}-}^{\alpha} f(a)+J_{\sqrt{a b}+}^{\alpha} f(b)\right] \mid \\
& \leq \frac{2^{\alpha-1} M}{3(\ln (b / a))^{\alpha}} \\
& \quad \times\left\{\frac{b-a}{2^{\alpha}}\left(\ln \frac{b}{a}\right)^{\alpha}\right. \\
& \left.-\alpha\left[\int_{\sqrt{a b}}^{b}\left(\ln \frac{b}{t}\right)^{\alpha-1} d t-\int_{a}^{\sqrt{a b}}\left(\ln \frac{t}{a}\right)^{\alpha-1} d t\right]\right\} .
\end{aligned}
$$

Specially, if we take $\alpha=1$ in this inequality, then we have

$$
\begin{aligned}
& \left|\frac{1}{3}\left[\frac{f(a)+f(b)}{2}+2 f(\sqrt{a b})\right]-\frac{1}{\ln b-\ln a} \int_{a}^{b} \frac{f(t)}{t} d t\right| \\
& \leq \frac{M}{3(\ln b-\ln a)}\left\{\frac{b-a}{2}\left(\ln \frac{b}{a}\right)-(a+b-2 \sqrt{a b})\right\} .
\end{aligned}
$$

(2) If we take $x=\sqrt{a b}$ and $\lambda=1 / 2$, then

$$
\begin{aligned}
& \mid \frac{1}{2}\left[\frac{f(a)+f(b)}{2}+2 f(\sqrt{a b})\right] \\
& -\frac{2^{\alpha-1} \Gamma(\alpha+1)}{(\ln (b / a))^{\alpha}}\left[J_{\sqrt{a b}-}^{\alpha} f(a)+J_{\sqrt{a b}+}^{\alpha} f(b)\right] \mid \\
& \leq \frac{M(b-a)}{4} .
\end{aligned}
$$

Specially, if we take $\alpha=1$ in this inequality, then we have

$$
\begin{aligned}
& \left|\frac{1}{2}\left[\frac{f(a)+f(b)}{2}+2 f(\sqrt{a b})\right]-\frac{1}{\ln b-\ln a} \int_{a}^{b} \frac{f(t)}{t} d t\right| \\
& \leq \frac{M(b-a)}{4} .
\end{aligned}
$$

Corollary 12. In Theorem 8 , If we take $\alpha=1$, then

$$
\begin{aligned}
& \mid(1-\lambda) f(x)+\lambda\left[\frac{f(a) \ln (x / a)+f(b) \ln (b / x)}{\ln b-\ln a}\right] \\
& -\frac{1}{\ln b-\ln a} \int_{a}^{b} \frac{f(t)}{t} d t \mid \\
& \leq \frac{M}{\ln b-\ln a}\left\{[(1-\lambda) x-\lambda a]\left(\ln \frac{x}{a}\right)\right. \\
& +[\lambda b-(1-\lambda) x]\left(\ln \frac{b}{x}\right) \\
& +(1-2 \lambda)(a+b-2 x)\} .
\end{aligned}
$$

Specially, if we take $x=\sqrt{a b}$ in this inequality, then we have

$$
\begin{aligned}
& \mid(1-\lambda) f(\sqrt{a b})+\lambda\left(\frac{f(a)+f(b)}{2}\right) \\
& -\frac{1}{\ln b-\ln a} \int_{a}^{b} \frac{f(t)}{t} d t \mid \\
& \leq \frac{M}{\ln b-\ln a} \\
& \quad \times\left[\frac{\lambda(b-a)}{2} \ln \frac{b}{a}+(1-2 \lambda)(a+b-2 \sqrt{a b})\right] .
\end{aligned}
$$

We note that if we take $\lambda=0, \lambda=1, \lambda=1 / 3$, and $\lambda=1 / 2$ in inequality (25) we obtain inequalities (16), (19), (21), and (23), respectively.

Let $f: I \subseteq(0, \infty) \rightarrow \mathbb{R}$ be an $M$-Lipschitzian function. In the next theorem, let $\lambda \in[0,1], C=a^{1-\lambda} b^{\lambda}, x, y \in[a, b]$ and define $C_{\alpha, \lambda}, \alpha>0$ as follows.

(1) If $a \leq C \leq x \leq y \leq b$, then

$$
\begin{aligned}
C_{\alpha, \lambda}(x, y)= & \frac{x}{\alpha} \lambda^{\alpha}\left(\ln \frac{b}{a}\right)^{\alpha} \\
& +\frac{y}{\alpha}\left\{(1-\lambda)^{\alpha}\left(\ln \frac{b}{a}\right)^{\alpha}-2\left(\ln \frac{b}{y}\right)^{\alpha}\right\} \\
& +\int_{y}^{b}\left(\ln \frac{b}{t}\right)^{\alpha-1} d t-\int_{C}^{y}\left(\ln \frac{b}{t}\right)^{\alpha-1} d t \\
& -\int_{a}^{C}\left(\ln \frac{t}{a}\right)^{\alpha-1} d t .
\end{aligned}
$$


(2) If $a \leq x \leq C \leq y \leq b$, then

$$
\begin{aligned}
C_{\alpha, \lambda}(x, y)= & \frac{x}{\alpha}\left\{2\left(\ln \frac{x}{a}\right)^{\alpha}-\lambda^{\alpha}\left(\ln \frac{b}{a}\right)^{\alpha}\right\} \\
& +\frac{y}{\alpha}\left\{(1-\lambda)^{\alpha}\left(\ln \frac{b}{a}\right)^{\alpha}-2\left(\ln \frac{b}{y}\right)^{\alpha}\right\} \\
& +\int_{x}^{C}\left(\ln \frac{t}{a}\right)^{\alpha-1} d t-\int_{a}^{x}\left(\ln \frac{t}{a}\right)^{\alpha-1} d t \\
& +\int_{y}^{b}\left(\ln \frac{b}{t}\right)^{\alpha-1} d t-\int_{C}^{y}\left(\ln \frac{b}{t}\right)^{\alpha-1} d t .
\end{aligned}
$$

(3) If $a \leq x \leq y \leq C \leq b$, then

$$
\begin{aligned}
C_{\alpha, \lambda}(x, y)= & \frac{x}{\alpha}\left\{2\left(\ln \frac{x}{a}\right)^{\alpha}-\lambda^{\alpha}\left(\ln \frac{b}{a}\right)^{\alpha}\right\} \\
& -\frac{y}{\alpha}(1-\lambda)^{\alpha}\left(\ln \frac{b}{a}\right)^{\alpha}+\int_{C}^{b}\left(\ln \frac{b}{t}\right)^{\alpha-1} d t \\
& +\int_{x}^{C}\left(\ln \frac{t}{a}\right)^{\alpha-1} d t-\int_{a}^{x}\left(\ln \frac{t}{a}\right)^{\alpha-1} d t .
\end{aligned}
$$

Now, we shall give another result for Lipschitzian functions as follows.

Theorem 13. Let $x, y, \alpha, \lambda, C, C_{\alpha, \lambda}$ and function $f$ be defined as above. Then we have the following inequality for Hadamard fractional integrals:

$$
\left|S_{f}(x, y, \lambda, \alpha, a, b)\right| \leq \frac{\alpha M C_{\alpha, \lambda}(x, y)}{(\ln (b / a))^{\alpha}} .
$$

Proof. Using the hypothesis of $f$, we have the following inequality:

$$
\begin{aligned}
& \left|S_{f}(x, y, \lambda, \alpha, a, b)\right| \\
& =\frac{\alpha}{(\ln (b / a))^{\alpha}} \mid \int_{a}^{C} \frac{[f(x)-f(t)]}{t}\left(\ln \frac{t}{a}\right)^{\alpha-1} d t \\
& +\int_{C}^{b} \frac{[f(y)-f(t)]}{t}\left(\ln \frac{b}{t}\right)^{\alpha-1} d t \mid \\
& \leq \frac{\alpha}{(\ln (b / a))^{\alpha}}\left[\int_{a}^{C} \frac{|f(x)-f(t)|}{t}\left(\ln \frac{t}{a}\right)^{\alpha-1} d t\right. \\
& \left.+\int_{C}^{b} \frac{f|(y)-f(t)|}{t}\left(\ln \frac{b}{t}\right)^{\alpha-1} d t\right] \\
& \leq \frac{\alpha M}{(\ln (b / a))^{\alpha}}\left[\int_{a}^{C} \frac{|x-t|}{t}\left(\ln \frac{t}{a}\right)^{\alpha-1} d t\right. \\
& \left.+\int_{C}^{b} \frac{|y-t|}{t}\left(\ln \frac{b}{t}\right)^{\alpha-1} d t\right] .
\end{aligned}
$$

Now, using simple calculations, we obtain the following identities $\int_{a}^{C}(|x-t| / t)(\ln (t / a))^{\alpha-1} d t$ and $\int_{C}^{b}(\mid y-$ $t \mid / t)(\ln (b / t))^{\alpha-1} d t$.
(1) If $a \leq C \leq x \leq y \leq b$, then

$$
\begin{aligned}
& \int_{a}^{C} \frac{|x-t|}{t}\left(\ln \frac{t}{a}\right)^{\alpha-1} d t \\
& =\frac{x}{\alpha} \lambda^{\alpha}\left(\ln \frac{b}{\alpha}\right)^{\alpha}-\int_{a}^{C}\left(\ln \frac{t}{a}\right)^{\alpha-1} d t \\
& \int_{C}^{b} \frac{|y-t|}{t}\left(\ln \frac{b}{t}\right)^{\alpha-1} d t \\
& =\frac{y}{\alpha}\left\{(1-\lambda)^{\alpha}\left(\ln \frac{b}{a}\right)^{\alpha}-2\left(\ln \frac{b}{y}\right)^{\alpha}\right\} \\
& +\int_{y}^{b}\left(\ln \frac{b}{t}\right)^{\alpha-1} d t-\int_{C}^{y}\left(\ln \frac{b}{t}\right)^{\alpha-1} d t .
\end{aligned}
$$

(2) If $a \leq x \leq C \leq y \leq b$, then

$$
\begin{aligned}
& \int_{a}^{C} \frac{|x-t|}{t}\left(\ln \frac{t}{a}\right)^{\alpha-1} d t \\
&= \frac{x}{\alpha}\left\{2\left(\ln \frac{x}{a}\right)^{\alpha}-\lambda^{\alpha}\left(\ln \frac{b}{a}\right)^{\alpha}\right\} \\
&+\int_{x}^{C}\left(\ln \frac{t}{a}\right)^{\alpha-1} d t-\int_{a}^{x}\left(\ln \frac{t}{a}\right)^{\alpha-1} d t \\
& \int_{C}^{b} \frac{|y-t|}{t}\left(\ln \frac{b}{t}\right)^{\alpha-1} d t \frac{y}{\alpha}\left\{(1-\lambda)^{\alpha}\left(\ln \frac{b}{a}\right)^{\alpha}-2\left(\ln \frac{b}{y}\right)^{\alpha}\right\} \\
& \quad+\int_{y}^{b}\left(\ln \frac{b}{t}\right)^{\alpha-1} d t-\int_{C}^{y}\left(\ln \frac{b}{t}\right)^{\alpha-1} d t .
\end{aligned}
$$

(3) If $a \leq x \leq y \leq C \leq b$, then

$$
\begin{aligned}
& \int_{a}^{C} \frac{|x-t|}{t}\left(\ln \frac{t}{a}\right)^{\alpha-1} d t \\
& =\frac{x}{\alpha}\left\{2\left(\ln \frac{x}{a}\right)^{\alpha}-\lambda^{\alpha}\left(\ln \frac{b}{a}\right)^{\alpha}\right\} \\
& \quad+\int_{x}^{C}\left(\ln \frac{t}{a}\right)^{\alpha-1} d t-\int_{a}^{x}\left(\ln \frac{t}{a}\right)^{\alpha-1} d t \\
& \int_{C}^{b} \frac{|y-t|}{t}\left(\ln \frac{b}{t}\right)^{\alpha-1} d t \\
& =\int_{C}^{b}\left(\ln \frac{b}{t}\right)^{\alpha-1} d t-\frac{y}{\alpha}(1-\lambda)^{\alpha}\left(\ln \frac{b}{a}\right)^{\alpha} .
\end{aligned}
$$

Using inequality (30) and the above identities $\int_{a}^{C}(\mid x-$ $t \mid / t)(\ln (t / a))^{\alpha-1} d t$ and $\int_{C}^{b}(|y-t| / t)(\ln (b / t))^{\alpha-1} d t$, we derive inequality (29). This completes the proof.

Under the assumptions of Theorem 13, we have the following corollaries and remarks. 
Corollary 14. In Theorem 13 , if we take $\alpha=1$, then inequality (29) reduces the following inequality:

$$
\begin{aligned}
& \left|(1-\lambda) f(x)+\lambda f(y)-\frac{1}{\ln b-\ln a} \int_{a}^{b} \frac{f(t)}{t} d t\right| \\
& \quad \leq \frac{M C_{1, \lambda}(x, y)}{\ln (b / a)} .
\end{aligned}
$$

Corollary 15. In Theorem 13 , let $\delta \in[1 / 2,1], x=a^{\delta} b^{1-\delta}$, and $y=a^{1-\delta} b^{\delta}$. Then, we have the inequality

$$
\begin{aligned}
& \mid \lambda^{\alpha} f\left(a^{\delta} b^{1-\delta}\right)+(1-\lambda)^{\alpha} f\left(a^{1-\delta} b^{\delta}\right) \\
& -\frac{\Gamma(\alpha+1)}{(\ln (b / a))^{\alpha}}\left[J_{C-}^{\alpha} f(a)+J_{C+}^{\alpha} f(b)\right] \mid \\
& \leq M L(\alpha, \lambda, \delta),
\end{aligned}
$$

as follows.

(i) If $\lambda \leq 1-\delta$, then

$$
\begin{aligned}
& L(\alpha, \lambda, \delta) \\
& =a^{\delta} b^{1-\delta} \lambda^{\alpha}+a^{1-\delta} b^{\delta}\left[(1-\lambda)^{\alpha}-2(1-\delta)^{\alpha}\right] \\
& +\frac{\alpha}{(\ln (b / a))^{\alpha}} \\
& \times\left\{\int_{a^{1-\delta} b^{\delta}}^{b}\left(\ln \frac{b}{t}\right)^{\alpha-1} d t\right. \\
& \left.-\int_{C}^{a^{1-\delta} b^{\delta}}\left(\ln \frac{b}{t}\right)^{\alpha-1} d t-\int_{a}^{C}\left(\ln \frac{t}{a}\right)^{\alpha-1} d t\right\}
\end{aligned}
$$

(ii) If $1-\delta \leq \lambda \leq \delta$, then

$L(\alpha, \lambda, \delta)$

$$
\begin{aligned}
= & a^{\delta} b^{1-\delta}\left[2(1-\delta)^{\alpha}-\lambda^{\alpha}\right]+a^{1-\delta} b^{\delta} \\
& \times\left[(1-\lambda)^{\alpha}-2(1-\delta)^{\alpha}\right]+\frac{\alpha}{(\ln (b / a))^{\alpha}} \\
& \times\left\{\int_{a^{\delta} b^{1-\delta}}^{C}\left(\ln \frac{t}{a}\right)^{\alpha-1} d t-\int_{a}^{a^{\delta} b^{1-\delta}}\left(\ln \frac{t}{a}\right)^{\alpha-1} d t\right. \\
& \left.+\int_{a^{1-\delta} b^{\delta}}^{b}\left(\ln \frac{b}{t}\right)^{\alpha-1} d t-\int_{C}^{a^{1-\delta} b^{\delta}}\left(\ln \frac{b}{t}\right)^{\alpha-1} d t\right\} .
\end{aligned}
$$

(iii) If $\delta \leq \lambda$, then

$$
L(\alpha, \lambda, \delta)
$$

$$
\begin{aligned}
=a^{\delta} b^{1-\delta}\left[2(1-\delta)^{\alpha}-\lambda^{\alpha}\right]-a^{1-\delta} b^{\delta}(1-\lambda)^{\alpha} \\
+\frac{\alpha}{(\ln (b / a))^{\alpha}}\left\{\int_{C}^{b}\left(\ln \frac{b}{t}\right)^{\alpha-1} d t\right. \\
+\int_{a^{\delta} b^{1-\delta}}^{C}\left(\ln \frac{t}{a}\right)^{\alpha-1} d t \\
\left.-\int_{a}^{a^{\delta} b^{1-\delta}}\left(\ln \frac{t}{a}\right)^{\alpha-1} d t\right\} .
\end{aligned}
$$

Corollary 16. In Theorem 13 , if we take $x=y=C$, then we have the inequality

$$
\begin{gathered}
\left|\left[\lambda^{\alpha}+(1-\lambda)^{\alpha}\right] f(x)-\frac{\Gamma(\alpha+1)}{(\ln (b / a))^{\alpha}}\left[J_{x-}^{\alpha} f(a)+J_{x+}^{\alpha} f(b)\right]\right| \\
\leq M\left\{\left[\lambda^{\alpha}-(1-\lambda)^{\alpha}\right] x+\frac{\alpha}{(\ln (b / a))^{\alpha}}\right. \\
\left.\times\left[\int_{x}^{b}\left(\ln \frac{b}{t}\right)^{\alpha-1} d t-\int_{a}^{x}\left(\ln \frac{t}{a}\right)^{\alpha-1} d t\right]\right\} .
\end{gathered}
$$

Remark 17. In inequality (39), if we choose $\lambda=1 / 2, C=\sqrt{a b}$, then we get inequality (15).

Corollary 18. In inequality (35), if we take $\delta=1$, then we have the following weighted Hadamard-type inequalities for Lipschitzian functions via Hadamard fractional integrals:

$$
\begin{gathered}
\left|\lambda^{\alpha} f(a)+(1-\lambda)^{\alpha} f(b)-\frac{\Gamma(\alpha+1)}{(\ln (b / a))^{\alpha}}\left[J_{C-}^{\alpha} f(a)+J_{C+}^{\alpha} f(b)\right]\right| \\
\leq M\left\{b(1-\lambda)^{\alpha}-a \lambda^{\alpha}+\frac{\alpha}{(\ln (b / a))^{\alpha}}\right. \\
\left.\times\left[\int_{a}^{C}\left(\ln \frac{t}{a}\right)^{\alpha-1} d t-\int_{C}^{b}\left(\ln \frac{b}{t}\right)^{\alpha-1} d t\right]\right\} .
\end{gathered}
$$

Remark 19. In inequality (40), if we choose $\lambda=1 / 2, C=$ $\sqrt{a b}$, then we get inequality (18).

\section{Application to Special Means}

Let us recall the following special means of two positive numbers $a, b$ with $b>a$.

(1) The arithmetic mean:

$$
A=A(a, b):=\frac{a+b}{2} .
$$

(2) The geometric mean:

$$
G=G(a, b):=\sqrt{a b} .
$$


(3) The harmonic mean:

$$
H=H(a, b):=\frac{2 a b}{a+b} .
$$

(4) The logarithmic mean:

$$
L=L(a, b):=\frac{b-a}{\ln b-\ln a} .
$$

(5) The identric mean:

$$
I=I(a, b)=\frac{1}{e}\left(\frac{b^{b}}{a^{a}}\right)^{1 /(b-a)}
$$
lemma.

To prove the results of this section, we need the following

Lemma 20 (see [12]). Let $f:[a, b] \rightarrow \mathbb{R}$ be differentiable with $\left\|f^{\prime}\right\|_{\infty}<\infty$. Then $f$ is an M-Lipschitzian function on $[a, b]$, where $M=\left\|f^{\prime}\right\|_{\infty}$.

Proposition 21. For $b>a>0, \lambda \in[0,1]$ and $n \geq 1$, we have

$$
\begin{aligned}
& \mid(1-\lambda) G^{n}(a, b)+\lambda A\left(a^{n}, b^{n}\right)-L\left(a^{n}, b^{n}\right) \mid \\
& \leq \frac{n b^{n-1}}{\ln b-\ln a} \\
& \quad \times\left[\frac{\lambda(b-a)}{2} \ln \frac{b}{a}+2(1-2 \lambda)(A(a, b)-G(a, b))\right] .
\end{aligned}
$$

Proof. The proof follows by inequality (25) applied for the Lipschitzian function $f(x)=x^{n}$ on $[a, b]$.

Remark 22. Let $\lambda=0$ and $\lambda=1$ in inequality (46). Then, using inequality (9), we have the following inequalities, respectively,

$$
\begin{aligned}
0 & \leq L\left(a^{n}, b^{n}\right)-G^{n}(a, b) \\
& \leq \frac{2 n b^{n-1}}{\ln b-\ln a}(A(a, b)-G(a, b)), \\
0 & \leq A\left(a^{n}, b^{n}\right)-L\left(a^{n}, b^{n}\right) \\
& \leq \frac{n b^{n-1}}{\ln b-\ln a}\left[\frac{b-a}{2} \ln \frac{b}{a}-2(A(a, b)-G(a, b))\right] .
\end{aligned}
$$

Proposition 23. For $b>a>0$ and $\lambda \in[0,1]$, we have

$$
\begin{aligned}
& \mid(1-\lambda) G\left(a e^{a}, b e^{b}\right) \lambda A\left(a e^{a}, b e^{b}\right) \\
&-L\left(e^{a}, e^{b}\right) L(a, b) \mid \\
& \leq \frac{e^{b}(b+1)}{\ln b-\ln a}\left[\frac{\lambda(b-a)}{2} \ln \frac{b}{a}+2(1-2 \lambda)\right. \\
&\times(A(a, b)-G(a, b))] .
\end{aligned}
$$

Proof. The proof follows by inequality (25) applied for the Lipschitzian function $f(x)=x e^{x}$ on $[a, b]$.

Remark 24. Let $\lambda=0$ and $\lambda=1$ in inequality (49). Then, using inequality (9), we have the following inequalities, respectively,

$$
\begin{aligned}
0 & \leq L\left(e^{a}, e^{b}\right) L(a, b)-G\left(a e^{a}, b e^{b}\right) \\
& \leq \frac{2 e^{b}(b+1)}{\ln b-\ln a}(A(a, b)-G(a, b)), \\
0 & \leq A\left(a e^{a}, b e^{b}\right)-L\left(e^{a}, e^{b}\right) L(a, b) \\
& \leq \frac{e^{b}(b+1)}{\ln b-\ln a}\left[\frac{b-a}{2} \ln \frac{b}{a}-2(A(a, b)-G(a, b))\right] .
\end{aligned}
$$

Proposition 25. For $b>a>0, \lambda \in[0,1]$, and $n \geq 1$, we have

$$
\begin{aligned}
\left|(1-\lambda) G^{-1}(a, b)+\lambda H^{-1}(a, b)-L(a, b) G^{-2}(a, b)\right| \\
\leq \frac{1}{a^{2}(\ln b-\ln a)} \\
\quad \times\left[\frac{\lambda(b-a)}{2} \ln \frac{b}{a}+2(1-2 \lambda)(A(a, b)-G(a, b))\right] .
\end{aligned}
$$

Proof. The proof follows by inequality (25) applied for the Lipschitzian function $f(x)=1 / x$ on $[a, b]$.

Remark 26. Let $\lambda=0$ and $\lambda=1$ in inequality (51). Then, using inequality (9), we have the following inequalities, respectively,

$$
\begin{aligned}
0 \leq & L(a, b)-G(a, b) \\
\leq & \frac{G^{2}(a, b)}{a^{2}(\ln b-\ln a)}(A(a, b)-G(a, b)), \\
0 \leq & G^{2}(a, b)-L(a, b) H(a, b) \\
\leq & \frac{G^{2}(a, b) H(a, b)}{a^{2}(\ln b-\ln a)} \\
& \times\left[\frac{b-a}{2} \ln \frac{b}{a}-2(A(a, b)-G(a, b))\right] .
\end{aligned}
$$

Proposition 27. For $b>a>0$ and $\lambda \in[0,1]$, we have

$$
\begin{aligned}
\ln G(a, b) \leq & \frac{1}{a(\ln b-\ln a)} \\
& \times\left[\frac{\lambda(b-a)}{2} \ln \frac{b}{a}+2(1-2 \lambda)\right. \\
& \times(A(a, b)-G(a, b))] .
\end{aligned}
$$

Proof. The proof follows by inequality (25) applied for the Lipschitzian function $f(x)=\ln x$ on $[a, b]$. 
Proposition 28. For $b>a>e^{-1}$ and $\lambda \in[0,1]$, we have

$$
\begin{aligned}
& \mid(1-\lambda) G(a, b) \ln G(a, b)+\lambda \ln G\left(a^{a}, b^{b}\right) \\
& -L(a, b) \ln I(a, b) \mid \\
& \leq \frac{1+\ln b}{\ln b-\ln a}\left[\frac{\lambda(b-a)}{2} \ln \frac{b}{a}+2(1-2 \lambda)\right. \\
& \times(A(a, b)-G(a, b))] .
\end{aligned}
$$

Proof. The proof follows by inequality (25) applied for Lipschitzian function $f(x)=x \ln x$ on $[a, b]$.

\section{Conflict of Interests}

The author declares that there is no conflict of interests regarding the publication of this paper.

\section{References}

[1] M. Alomari, M. Darus, S. S. Dragomir, and P. Cerone, "Ostrowski type inequalities for functions whose derivatives are s-convex in the second sense," Applied Mathematics Letters, vol. 23, no. 9, pp. 1071-1076, 2010.

[2] M. Avci, H. Kavurmaci, and M. E. Özdemir, "New inequalities of Hermite-Hadamard type via $s$-convex functions in the second sense with applications," Applied Mathematics and Computation, vol. 217, no. 12, pp. 5171-5176, 2011.

[3] Y. Chu, G. Wang, and X. Zhang, "Schur convexity and hadamard's inequality," Mathematical Inequalities and Applications, vol. 13, no. 4, pp. 725-731, 2010.

[4] İ. İşcan, "A new generalization of some integral inequalities for $(\alpha, m)$-convex functions," Mathematical Sciences, vol. 7, no. 22, pp. 1-8, 2013.

[5] İ. İşcan, "New estimates on generalization of some integral inequalities for $(\alpha, m)$-convex functions," Journal of Contemporary Applied Mathematics, vol. 1, no. 2, pp. 253-264, 2013.

[6] İ. İşcan, "New estimates on generalization of some integral inequalities for s-convex functions and their applications," International Journal of Pure and Applied Mathematics, vol. 86, no. 4, pp. 727-746, 2013.

[7] E. Set, M. E. Ozdemir, and M. Z. Sarkıaya, "On new inequalities of Simpson's type for quasiconvex functions with applications," Tamkang Journal of Mathematics, vol. 43, no. 3, pp. 357-364, 2012.

[8] M. Z. Sarkıaya, E. Set, and M. E. Ozdemir, "On new inequalities of Simpson's type for s-convex functions," Computers \& Mathematics with Applications, vol. 60, pp. 2191-2199, 2010.

[9] Y.-M. Chu, X.-M. Zhang, and X.-H. Zhang, "The hermitehadamard type inequality of GA-convex functions and its application," Journal of Inequalities and Applications, vol. 2010, Article ID 507560, 11 pages, 2010.

[10] S. S. Dragomir, Y. J. Cho, and S. S. Kim, "Inequalities of Hadamard's type for Lipschitzian mappings and their applications," Journal of Mathematical Analysis and Applications, vol. 245, no. 2, pp. 489-501, 2000.

[11] S.-R. Hwang, K.-C. Hsu, and K.-L. Tseng, "Hadamard-type inequalities for Lipschitzian functions in one and two variables with applications," Journal of Mathematical Analysis and Applications, vol. 405, no. 2, pp. 546-554, 2013.
[12] K.-L. Tseng, S.-R. Hwang, and K.-C. Hsu, "Hadamard-type and Bullen-type inequalities for Lipschitzian functions and their applications," Computers and Mathematics with Applications, vol. 64, no. 4, pp. 651-660, 2012.

[13] G.-S. Yang and K.-L. Tseng, "Inequalities of hadamard's type for Lipschitzian mappings," Journal of Mathematical Analysis and Applications, vol. 260, no. 1, pp. 230-238, 2001.

[14] C. P. Niculescu, "Convexity according to the geometric mean," Mathematical Inequalities and Applications, vol. 3, no. 2, pp. 155167,2000

[15] C. P. Niculescu, "Convexity according to means," Mathematical Inequalities and Applications, vol. 6, no. 4, pp. 571-579, 2003.

[16] A. A. Kilbas, H. M. Srivastava, and J. J. Trujillo, Theory and Applications of Fractional Differential Equations, Elsevier B.V., Amsterdam, The Netherlands, 2006.

[17] İ. İşcan, "New general integral inequalities for quasi-geometrically convex functions via fractional integrals," Journal of Inequalities and Applications, vol. 2013, no. 491, pp. 1-15, 2013. 


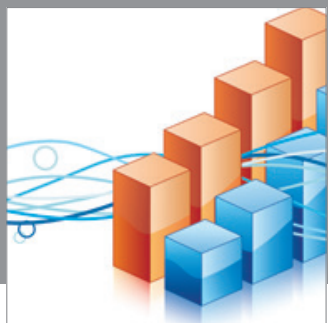

Advances in

Operations Research

mansans

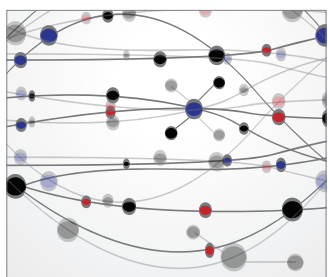

The Scientific World Journal
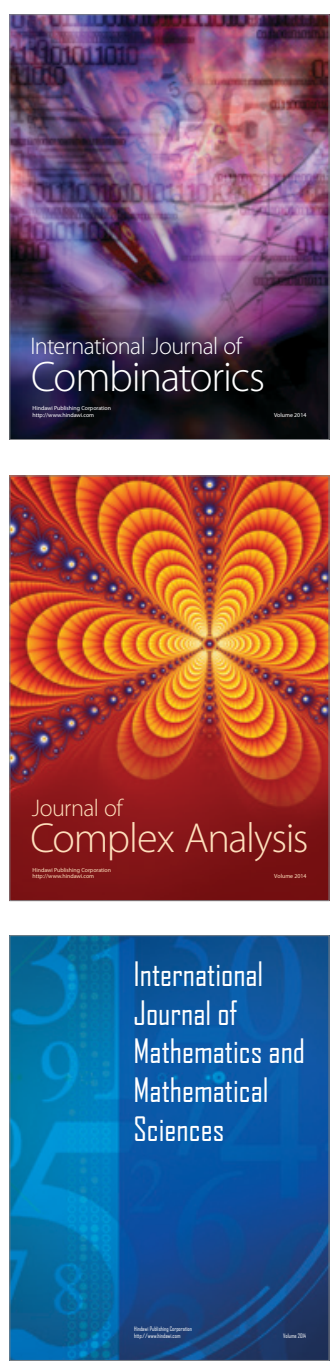
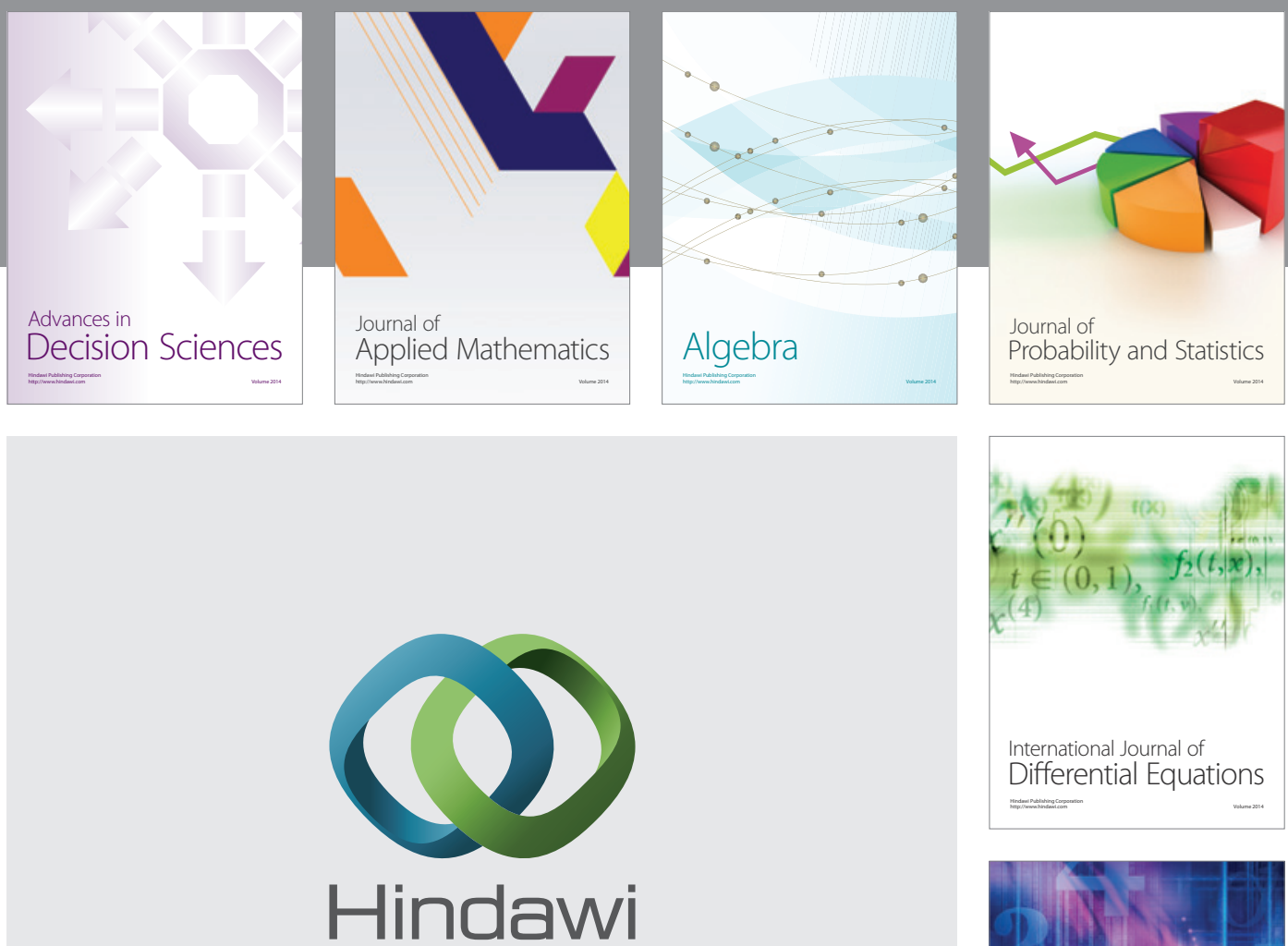

Submit your manuscripts at http://www.hindawi.com
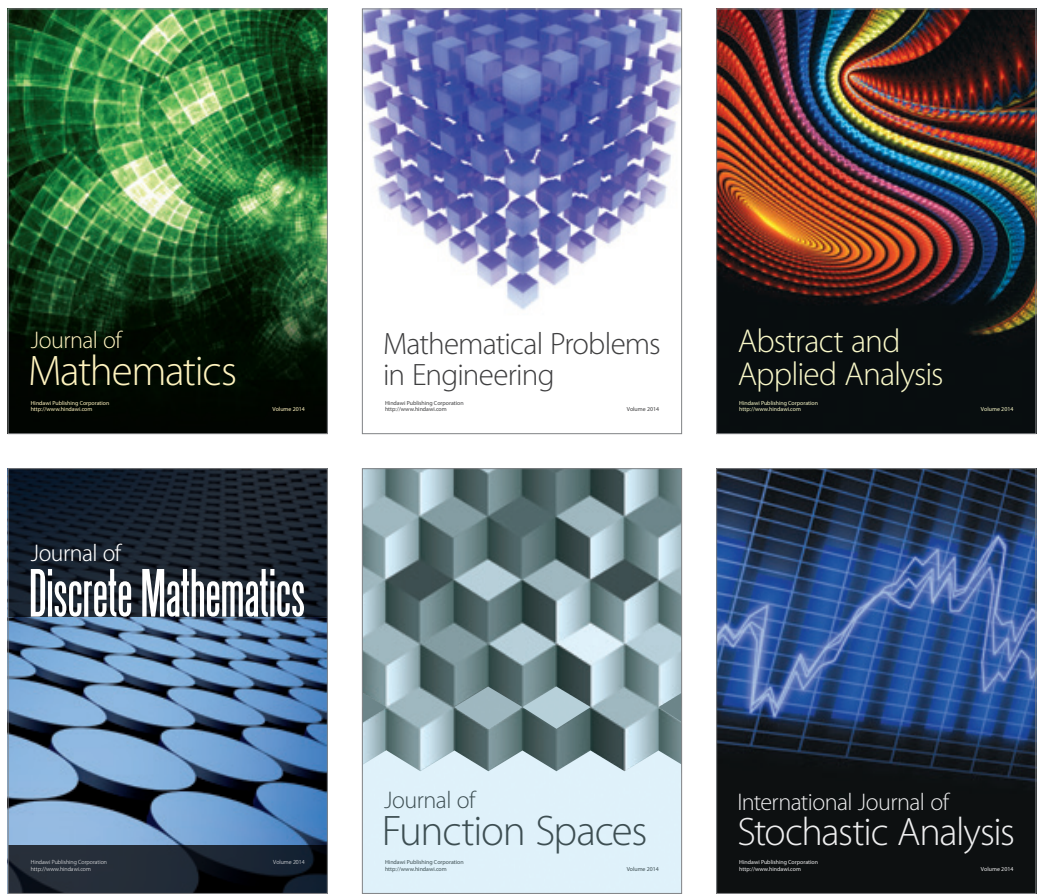

Journal of

Function Spaces

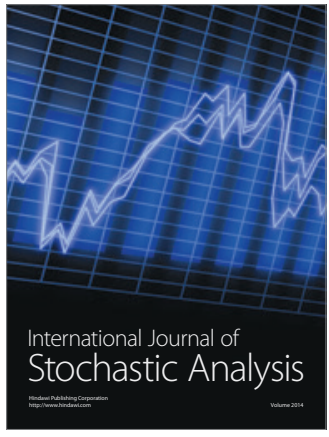

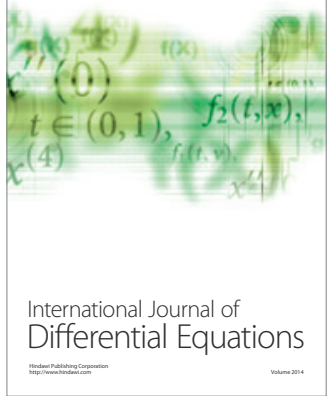
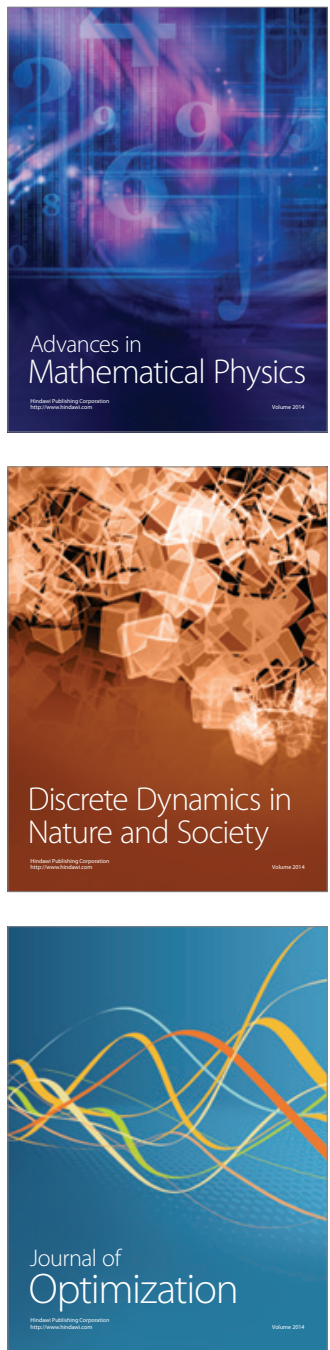